УДК 519.68

DOI: $10.15827 / 0236-235 X .123 .557-564$

Дата подачи статьи: 15.02 .18

\title{
Эфрфективность поэтапного применения средств защиты с пересечением областей обнаружения угроз
}

\author{
B.C. Коломойцев 1, аспирант, dek-s-kornis@yandex.ru \\ B.A. Богатырев 1, д.m.н., npodeccop, vladimir.bogatyrev@gmail.com
}

1 Санкт-Петербургский наииональный исследовательский университет информаиионных технологий, механики и оптики (Университет ИТМО), г. Санкт-Петербург, 197101, Россия

В работе исследованы возможности повышения эффективности схемы безопасного доступа «Прямое соединение» на основе выбора вариантов ее построения с учетом поэтапного применения различных средств защиты информации и их объединения в систему кластерной архитектуры, направленного на консолидацию ограниченных ресурсов защиты. Консолидация ресурсов защиты проводится с целью достижения максимальной защищенности при минимальных задержках и стоимости системы.

Предложены критерии эффективности систем защиты информации, ориентированные на повышение вероятности обнаружения угроз за минимальное время, с учетом его возможной ограниченности. Данные критерии учитывают среднее время пребывания запросов и вероятности обнаружения и устранения угроз системой защиты информации. Время обслуживания на каждом этапе предполагается распределенным по показательному закону.

Предложены модели оценки вероятности обнаружения угроз и задержек их поиска в зависимости от последовательности применения средств защиты информации в узлах. Модели учитывают пересекаемость множеств угроз, обнаруживаемых различными средствами защиты информации. Показана возможность реализации адаптивной стратегии последовательного применения средств защиты информации для предотвращения угроз информационной безопасности в зависимости от изменяемой интенсивности поступающих запросов. Рассмотрены варианты комплектации узлов полным или частичным набором имеющихся программных или аппаратно-программных средств защиты информации.

Ключевые слова: информационная безопасность, защита информации, схема доступа, несанкиионированный доступ, оиенка эффективности системы, системы массового обслуживания, вероятность обнаружения угроз, информачионные угрозы.

Обеспечение высокой надежности и информационной защищенности является одной из ключевых задач в современных системах обработки, хранения и передачи информации. Угрозы информационной безопасности (ИБ) могут иметь многообразные проявления, например, в виде вирусных атак, направленных на искажение или уничтожение обрабатываемых в вычислительной системе данных, или несанкционированного доступа к узлам вычислительной сети [1-4].

Актуальными представляются исследования влияния на эффективность вычислительной системы организации системы защиты информации (ЗИ) и схемы безопасного доступа (СБД), позволяющих безопасно взаимодействовать со всеми внешними и внутренними узлами инфокоммуникационной системы [5].

Проектирование системы ЗИ для сложных инфокоммуникационных систем требует модельноориентированного подхода, при котором предполагается оценка эффективности проектных решений по построению системы ЗИ рациональных проектных решений с их дальнейшей оптимизацией. Выбор и оптимизация проектных решений требуют формирования системы критериев эффективности системы ЗИ, включающей совокупность частных и комплексных критериев.

Цель работы - исследование возможностей повышения эффективности инфокоммуникационной системы, использующей СБД «Прямое соедине- ние», на основе выбора вариантов ее организации с учетом поэтапного применения различных средств ЗИ и их объединения в систему кластерной архитектуры, направленного на консолидацию ограниченных ресурсов защиты для достижения максимальной защищенности при минимальных задержках и стоимости системы.

\section{Объект исследования}

Рассматривается СБД «Прямое соединение» (а именно: ее частный случай - СБД «Связующий узел»), предполагающая совместное использование некоторого множества последовательно подключенных элементов (средств) защиты [5].

Будем считать, что система ЗИ содержит один или некоторое множество узлов, объединенных в кластер. Узлы кластера комплектуются некоторым множеством средств защиты, реализованных программно, аппаратно или аппаратно-программно. При комплектовании узлов аппаратными или аппаратно-программными средствами ЗИ возможно их последовательное, параллельное или конвейерное использование. Конструктивно узел может быть реализован в виде компьютера со встраиваемыми платами средств ЗИ. При этом стоимость узла пропорциональна числу включенных в его состав средств ЗИ. При программной реализации средств ЗИ узел содержит компьютер, укомплектованный набором программных средств ЗИ (СБД «Связую- 
щий узел». Инициализация функционирования средств ЗИ реализуется последовательно, причем задается различный порядок их использования. При программной реализации средств ЗИ стоимость узла в основном определяется стоимостью компьютера.

Типовая СБД «Прямое соединение» представлена на рисунке 1.

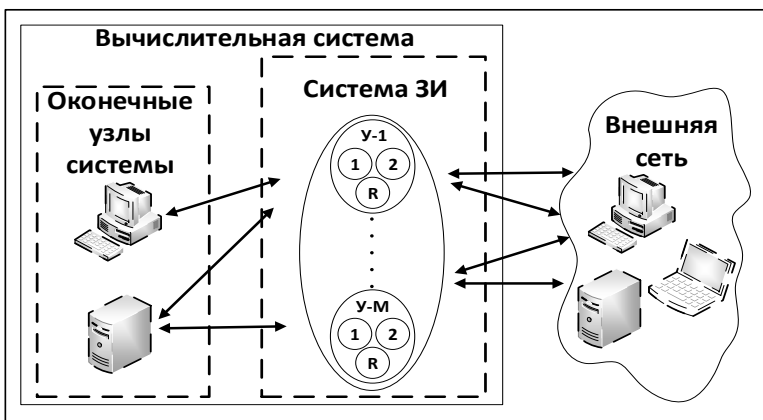

Рис. 1. Типовая СБД «Прямое соединение»

Fig. 1. A typical "Direct connection" PSA

Данная схема имеет три ключевые зоны:

- оконечные узлы вычислительной системы, содержащие встроенные персональные средства ЗИ;

- система ЗИ, представляющая набор средств ЗИ, встраиваемых в узлы и, возможно, объединяемых в кластер (кластер узлов ЗИ);

- внешняя инфокоммуникационная система объединяющая внешние (не подконтрольные системе) средства обработки, хранения и передачи данных.

В качестве базового решения рассмотрим систему ЗИ, реализуемую в виде вычислительного узла с некоторым набором программных средств защиты. Активизация работы используемых средств защиты осуществляется последовательно (поэтапно) с возможностью запуска в любом порядке. Каждый узел представим в виде одноканальной системы массового обслуживания (СМО) с общей бесконечной очередью и поэтапным выполнением запросов [6]. Процесс обслуживания запроса системой защиты, включающей $R$ этапов, представлен на рисунке 2.

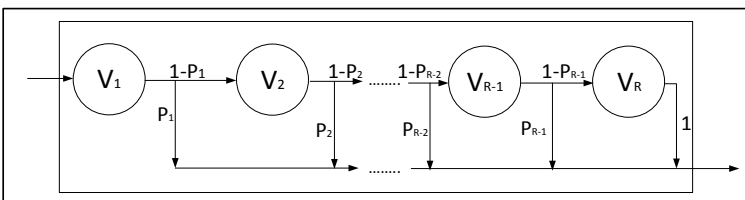

Рис. 2. Процесс обслужсивания запроса системой защитты, включаюший $R$ этапов: $V_{l}, \ldots, V_{R}-$ время обработки на этапах системы защиты; $P_{1}, \ldots, P_{R-1}-$ вероятность прохождения запросом $i$-го этапа системы защиты $, i=1, \ldots, R$

Fig. 2. The process of request maintenance by a security system that includes $R$-stages
После завершения очередного $i$-го этапа запрос либо с вероятностью $P_{i}$ немедленно покидает систему (средство обнаружило и устранило угрозу), либо с вероятностью $\left(1-P_{i}\right)$ поступает на следующий $(i+1)$-й этап обслуживания. После завершения обслуживания на этапе $R$ запрос покидает систему и начинается поэтапное выполнение следующего запроса из очереди. Время выполнения этапов будем считать распределенным по показательному закону [7].

\section{Критерии эффективности системы защиты}

К исследуемой системе защиты предъявляются требования высокой вероятности обнаружения отказов, малых задержек обнаружения угроз информационной безопасности и ограниченной (возможно, минимальной) стоимости системы зашиты.

Эффективность системы охарактеризуем некоторым набором частных и комплексных критериев (показателей). Набор критериев, характеризующих задержки поиска угроз, вероятность их обнаружения и надежность системы, по выполнению требуемых функций включает:

- среднее время обнаружения угрозы $(x)$;

- второй начальный момент $\left(x^{(2)}\right)$ распределения времени обнаружения угрозы;

- среднее время пребывания (ожидания) запросов $\left(T_{S}\right)$ в системе ЗИ во время поиска угроз;

- вероятность обнаружения и устранения угроз $i$-м элементом системы ЗИ $\left(P_{i}\right)$;

- вероятность обнаружения и устранения угроз системой ЗИ $\left(P_{S}\right)$;

- коэффициент готовности $\left(k_{\Gamma}\right)$;

- вероятность безотказной работы системы за время пребывания в ней запроса на обнаружение и устранение угрозы $\left(P\left(T_{S}\right)\right)$.

Рассмотрим комплексные показатели эффективности анализируемой системы.

Надежность системы ЗИ по обслуживанию запросов поиска угроз ИБ можно охарактеризовать по коэффициенту оперативной готовности $k_{\text {ог }}=$ $=k_{\Gamma} \cdot P\left(T_{S}\right)$, который является комплексным показателем и определяется вероятностью готовности системы ЗИ к обнаружению и устранению угроз в поступившем в систему запросе $\left(k_{\Gamma}\right)$ и безотказности задействованных при этом средств ЗИ за время пребывания запроса в системе ЗИ $\left(P\left(T_{S}\right)\right)$.

Задержку и вероятность обнаружения и устранения угроз системой ЗИ охарактеризуем по комплексному критерию - показатель эффективности системы ЗИ:

$$
Q_{s}=\frac{\left(T_{0}-T_{S}\right)}{T_{0}} P_{S},
$$

где $T_{0}$ - предельно допустимое время пребывания запроса в системе; $T_{S}$ - время пребывания запроса в системе; $P_{S}-$ вероятность обнаружения и устра- 
нения угроз системой ЗИ (степень защищенности вычислительной системы).

Предложенный критерий выражает нормированную среднюю экономию времени до обнаружения и устранения угрозы относительно предельно допустимого времени задержки, вносимой системой ЗИ. Критерий предполагает абсолютную надежность системы.

С учетом готовности системы ЗИ к решению задачи обнаружения угроз в произвольный момент времени критерий может быть модернизирован как

$$
Q_{s}=\frac{\left(T_{0}-T_{S}\right)}{T_{0}} k_{\mathrm{r}} P_{S},
$$

где $k_{\text {г }}$ - коэффициент готовности системы ЗИ (вероятность того, что в произвольный момент времени поступления запроса задачи поиска угроз система ЗИ работоспособна (готова к выполнению требуемой функции обнаружения и устранения угроз)).

Критерий может быть расширен с учетом не только готовности системы ЗИ в некоторый момент времени, но и вероятности безотказной работы системы в течение реализации процедуры обнаружения и устранения угроз:

$$
Q_{s}=\frac{\left(T_{0}-T_{S}\right)}{T_{0}} k_{\text {ог }} P_{S}=\frac{\left(T_{0}-T_{S}\right)}{T_{0}} k_{\mathrm{r}} P\left(T_{S}\right) P_{S},
$$

где $k_{\text {ог }}-$ коэффициент оперативной готовности; $P\left(T_{S}\right)$ - вероятность безотказной работы системы за время пребывания в ней запроса на обнаружение и устранение угрозы.

\section{Оценка задержек системы ЗИ}

СМО с поэтапным обслуживанием (рис. 2) является частным случаем СМО типа $M / G / 1$ [6]. Среднее время пребывания запроса в системе $T$ можно определить по формуле Полячика-Хинчина:

$$
T=\bar{x}+\frac{\lambda x^{(2)}}{2(1-\lambda \bar{x})},
$$

где $\bar{x}-$ среднее время обслуживания запроса; $x^{(2)}$ второй начальный момент; $\lambda$ - интенсивность входного потока [6].

Если СБД [8-13] включает $M$ систем, которые ведут обслуживание поступающих запросов, то интенсивность потока запросов, поступающих в каждую из $M$ систем, будет $\lambda / M$, а среднее время пребывания запросов в системе находиться как

$$
T_{S}=\bar{x}+\frac{\lambda x^{(2)}}{2(M-\lambda \bar{x})} .
$$

Определим первый и второй начальные моменты времени реализации $R$-этапной процедуры обнаружения и устранения угроз. Пусть время обслуживания каждого этапа - случайная величина, распределенная по показательному закону. В таком случае, используя распределение Кокса (в соответ- ствии с рисунком 2) [6], получаем преобразование Лапласа для плотности распределения вероятностей времени обслуживания в виде

$$
\begin{aligned}
& B(s)=\left(\frac{\mu_{1}}{s+\mu_{1}}\right) P_{1}+ \\
& +\left(\sum_{i=2}^{R-1} P_{i}\left(\prod_{j=1}^{I-1}\left(1-P_{j}\right)\right) \prod_{j=1}^{i}\left(\frac{\mu_{j}}{s+\mu_{j}}\right)+\right. \\
& +\prod_{i=1}^{R}\left(\frac{\mu_{i}}{s+\mu_{i}}\right)\left(\prod_{j=1}^{R-1}\left(1-P_{j}\right)\right),
\end{aligned}
$$

где $\mu_{i}$ и $P_{i}-$ интенсивность обслуживания и вероятность устранения угрозы на $i$-м этапе обслуживания; $R$ - количество этапов обслуживания (обнаружения и устранения угроз).

Для вычисления $n$-го начального момента случайной величины [6], воспользуемся следующей формулой: $\overline{X^{n}}=(-1)^{n} A^{*(n)}(0)$.

Первая производная $B(s)$ соответствует первому начальному моменту (математическому ожиданию) времени поэтапного поиска угрозы:

$$
x=\left.\frac{d B(s)}{d s}\right|_{s=0} .
$$

Вторая производная $B(s)$ соответствует второму начальному моменту распределения времени поэтапного поиска угрозы: $x^{(2)}=\left.\frac{d^{2} B(s)}{d s^{2}}\right|_{s=0}$

Для примера рассмотрим систему ЗИ вычислительной системы, включающей два элемента ЗИ $(R=2)$. В этом случае при двухэтапном обслуживании из формулы (6) получаем

$$
\begin{aligned}
& B^{\prime}(s)=\frac{\mu_{1} P_{1}}{\left(\mu_{1}+s\right)^{2}}+\frac{\mu_{1} \mu_{2}\left(1-P_{1}\right)}{\left(\mu_{1}+s\right)\left(\mu_{2}+s\right)^{2}}+ \\
& +\frac{\mu_{1} \mu_{2}\left(1-P_{1}\right)}{\left(\mu_{1}+s\right)^{2}\left(\mu_{2}+s\right)}, \\
& B^{\prime \prime}(s)=\frac{2 \cdot \mu_{1}}{\left(\mu_{1}+s\right)}\left(\frac{P_{1}}{\left(\mu_{1}+s\right)^{2}}+\frac{\mu_{2}\left(1-P_{1}\right)}{\left(\mu_{2}+s\right)^{3}}+\right. \\
& \left.+\frac{\mu_{2}\left(1-P_{1}\right)}{\left(\mu_{1}+s\right)\left(\mu_{2}+s\right)^{2}}+\frac{\mu_{2}\left(1-P_{1}\right)}{\left(\mu_{1}+s\right)^{2}\left(\mu_{2}+s\right)}\right) . \\
& \text { Отсюда } \\
& \bar{x}=V_{1} P_{1}+\left(1-P_{1}\right)\left(V_{1}+V_{2}\right) ; \\
& x^{(2)}=2\left(V_{1}^{2} P_{1}+V_{2}^{2}\left(1-P_{1}\right)+V_{1} V_{2}\left(1-P_{1}\right)+V_{1}^{2}\left(1-P_{1}\right)\right),
\end{aligned}
$$

где $V_{i}$ - среднее время обслуживания $i$-го средства ЗИ.

\section{Оценка вероятности обнаружения и устранения угроз}

Степень защищенности системы определим по вероятности обнаружения и устранения угрозы системой ЗИ [7].

Вероятность обнаружения угрозы после применения $r$ средств защиты при их независимости по 
областям обнаружения угроз можно определить как $P_{r}=1-\prod_{i=1}^{r}\left(1-p_{i}\right)$.

Определим вероятности совместного обнаружения и устранения угроз при пересекаемости множеств угроз, обнаруживаемых различными средствами ЗИ.

В общем виде пересечения множеств угроз ИБ для системы ЗИ, включающей три элемента, с указанием доли угроз ИБ, устраняемых каждым из элементов или группой элементов, показан диаграммой Венна на рисунке 3.

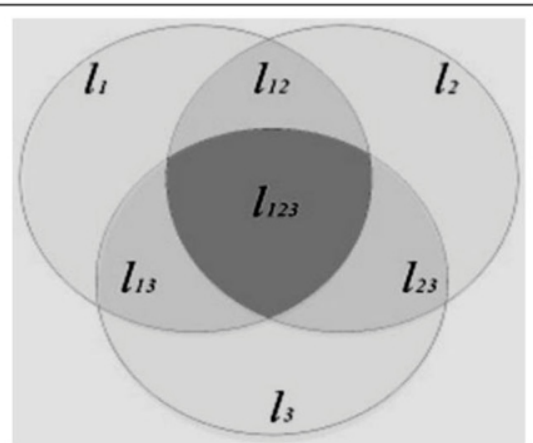

Рис. 3. Пересечения множеств угроз, устраняемых тремя средствами зашиты

Fig. 3. Crossings of sets of threats eliminated by three means of protection

Для характеристики зависимости обнаружения угроз используемыми средствами ЗИ определим:

- долю угроз от общего множества угроз ИБ, которые обнаруживаются и устраняются $i$-м элементом, $-L_{i}$;

- долю угроз от общего множества угроз ИБ, обнаруживаемых и устраняемых $i$-м элементом, применяемым в составе системы ЗИ, состоящей из $r$ элементов, $-l_{i}$;

- долю угроз от общего множества угроз ИБ, обнаруживаемых и устраняемых элементами $i, j$ (используя для этого отличные друг от друга методы и/или алгоритмы), применяемыми в составе системы ЗИ, состоящей из $r$ элементов, $-l_{i j}$;

- долю угроз от общего множества угроз ИБ, обнаруживаемых (и устраняемых) элементами $i, j$, $\ldots, m$ (используя для этого отличные друг от друга методы и/или алгоритмы), применяемыми в составе системы ЗИ, состоящей из $r$ элементов, $-l_{i j . . m}$;

- вероятность обнаружения системой ЗИ угроз ИБ, которым может быть подвержена вычислительная система, $-I_{S}$.

$$
L_{i}=\left|A_{i}\right| /|E| ; l_{i}=\frac{\left|A_{i} \backslash \bigcup_{\substack{j=1 \\ j \neq i}}^{r} A_{j}\right|}{|E|} ;
$$
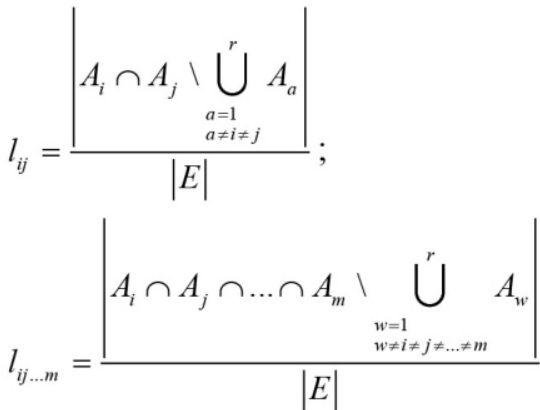

$I_{S}=|E| /|H|$,

где $|E|,|H|,\left|A_{i}\right|$ - кардинальные числа (число угроз) множеств, соответственно, $E, H, A_{i}$. При этом $H$ - с которыми необходимо бороться в рамках конкретной вычислительной системы; $E$ - множество угроз, которые способен обнаружить (и с некоторой вероятностью устранить) набор из $R$ средств (мер), использующихся в составе системы ЗИ; $A_{i}-$ множество угроз, которые способен обнаружить и устранить $i$-й элемент системы ЗИ.

Вероятность устранения и обнаружения угрозы $P_{S}$ при поэтапном применении средств защиты, вычислим как

$$
\begin{aligned}
& P_{S}=I_{S} W \sum_{i=1}^{R}\left(l_{i} p_{i}+\sum_{j=1}^{j<i} l_{j i} \cdot\left(1-\overline{p_{i}} \overline{p_{j}}\right)+\right. \\
& +\sum_{q=1}^{q<j}\left(l_{q j i} \cdot\left(1-\overline{p_{i}} \overline{p_{j}} \overline{p_{q}}\right)+\ldots+\right. \\
& \left.\ldots+\sum_{m=1}^{m<t}\left(l_{m t \ldots i} \cdot\left(1-\overline{p_{i}} \overline{p_{j}} \ldots \overline{p_{m}}\right)\right) \ldots\right),
\end{aligned}
$$

где $W=\lambda_{T} / \lambda$ - доля угроз ИБ в поступающем потоке запросов, здесь $\lambda_{T}$ и $\lambda$ - соответственно интенсивности входного потока угроз ИБ и общего потока запросов (в том числе с угрозами ИБ); $l_{i}-$ доля угроз ИБ от общего множества, определяемая только $i$-м элементом системы ЗИ, состоящей из $R$ элементов; $l_{i \ldots m}$ - доля угроз ИБ от общего множества угроз ИБ, обнаруживаемых и устраняемых элементами с $i, j$ по $m$, применяемыми в составе системы ЗИ, состоящей из нескольких элементов; $p_{i}-$ вероятность обнаружения угрозы $i$-м средством; $\overline{p_{j}}-$ вероятность, определяемая как $\overline{p_{j}}=1-p_{j}$; $i, j, \ldots, t$ - порядковые номера элементов системы ЗИ [7].

Используя (7), получим вероятность устранения угрозы $P_{i}$ на $i$-м этапе обслуживания [7]:

$$
\begin{aligned}
& P_{i}=I_{S} W \cdot\left(l_{i} p_{i}+\sum_{j=1}^{j<i}\left(l_{j i} \cdot\left(p_{i} \overline{p_{j}}\right)+\right.\right. \\
& \left.+\sum_{q=1}^{q<j}\left(\begin{array}{l}
l_{q j i} \cdot\left(p_{i} \overline{p_{j}} \overline{p_{q}}\right)+ \\
+\ldots+\sum_{m=1}^{m<t}\left(l_{m t \ldots i} \cdot\left(p_{i} \overline{p_{j}} \ldots \overline{p_{m}}\right)\right.
\end{array}\right)\right) \ldots .
\end{aligned}
$$


На основе (8) получим, что для системы, использующей два элемента защиты информации, $P_{1}=I_{S} W p_{1} L_{1}$, здесь $L_{1}=\left|A_{1}\right| /|E|$.

Используя (7), вероятность устранения и обнаружения угрозы системой $P_{S}$ вычислим как

$$
P_{S 2}=I_{S} W \cdot\left(p_{1} l_{1}+p_{2} l_{2}+l_{12} \cdot\left(1-\overline{p_{1}} \overline{p_{2}}\right)\right),
$$

здесь $l_{1}=L_{1}-l_{12.0} ; l_{2}=L_{2}-l_{12.0}$, где $l_{12.0}=$ $=\left|A_{1} \cap A_{2}\right| /|E|, L_{2}=\left|A_{2}\right| /|E|$.

\section{Обоснование выбора организации системы $3 И$}

В работах $[5,7]$ показано, что последовательное применение средств от слабых (быстрых и с малой областью обнаружения угроз) к наиболее сильным (имеющим большую область обнаружения угроз, но и затрачивающим большее количество времени на свою работу) дает лучшие результаты по среднему времени обнаружения угроз. Имея три средства ЗИ, можно выделить следующие варианты их последовательного применения: $(1,2,3) ;(1,2) ;(2$, 3); (1, 3); (1); (2); (3).

По формулам (3) и (4) получим, что для системы с последовательным применением трех средств ЗИ $(1,2,3)$ математическое ожидание и второй начальный момент среднего времени обслуживания будут равны

$$
\begin{aligned}
& \bar{x}=V_{1} P_{1}+\left(1-P_{1}\right) P_{2}\left(V_{1}+V_{2}\right)+ \\
& +\left(1-P_{1}\right)\left(1-P_{2}\right)\left(V_{1}+V_{2}+V_{3}\right), \\
& x^{(2)}=2\left(V_{1}^{2}\left(P_{1}+P_{2}\left(1-P_{1}\right)\right)+V_{2}^{2} P_{2}\left(1-P_{1}\right)+\right. \\
& \left.+V_{1} V_{2} P_{2}\left(1-P_{1}\right)+V_{3}^{2}\left(1-P_{1}\right)\left(1-P_{2}\right)\right)+ \\
& +\left(V_{1}^{2}+V_{1} V_{2}+V_{2}^{2}\right)\left(1-P_{1}\right)\left(1-P_{2}\right)+ \\
& +2 V_{3}\left(V_{1}+V_{2}\right)\left(1-P_{1}\right)\left(1-P_{2}\right) .
\end{aligned}
$$

Вероятность обнаружения и устранения угрозы системой при варианте $(1,2,3)$ получим как

$$
\begin{aligned}
& P_{S 3}=I_{S} W\left(p_{1} l_{1}+p_{2} l_{2}+p_{3} l_{3}+l_{12}\left(1-\overline{p_{1}} \overline{p_{2}}\right)+\right. \\
& +l_{23}\left(1-\overline{p_{2}} \overline{p_{3}}\right)+l_{13}\left(1-\overline{p_{1}} \overline{p_{3}}\right)+l_{123}\left(1-\overline{p_{1}} \overline{p_{2}} \overline{p_{3}}\right) .
\end{aligned}
$$

Вероятность обнаружения и предотвращения угрозы на первом и втором этапах контроля определяется как $P_{1}=I_{S} W p_{1} L_{1} ; \quad P_{2}=I_{S} W\left(p_{2}\left(L_{2}-l_{12}\right)+\right.$ $\left.\left.+l_{12}\left(1-p_{1}\right) p_{2}\right)\right)$.

Здесь $l_{1}=L_{1}-l_{12.0}-l_{13.0}+l_{123} ; l_{2}=L_{2}-l_{12.0}-l_{23.0}+$ $+l_{123} ; l_{3}=L_{3}-l_{13.0}-l_{23.0}+l_{123} ; l_{12}=L_{1}-l_{12.0}-l_{123}$; $l_{13}=l_{13.0}-l_{123} ; l_{23}=l_{23.0}-l_{123} ; l_{123}=l_{123.0}, L_{1}, L_{2}, L_{3}-$ доля угроз ИБ от общего множества, определяемых, соответственно, первым, вторым и третьим элементами системы ЗИ; $l_{12.0}=\left|A_{1} \cap A_{2}\right| /|E|, l_{23.0}=$ $=\left|A_{2} \cap A_{3}\right| /|E|, \quad l_{13.0}=\left|A_{1} \cap A_{3}\right| /|E|, \quad l_{123.0}=$ $=\left|A_{1} \cap A_{2} \cap A_{3}\right| /|E|-$ доля угроз ИБ от общего множества, определяемых, соответственно, первым и вторым, вторым и третьим, первым и третьим и первым, вторым и третьим элементами.

Для системы, использующей один элемент защиты, математическое ожидание и второй момент времени обнаружения угроз $\bar{x}=V_{1} P_{1} ; x^{(2)}=2 V_{1} P_{1}^{2}$.

Вероятность обнаружения и устранения угрозы на первом этапе обслуживания будет равна вероятности обнаружения и устранения угрозы системой и определяется как $P_{S 1}=P_{1}=I_{S} W p_{1} L_{1}$. Здесь $L_{1}=$ $=\left|A_{1}\right| /|E|$.

Для примера будем считать, что десятая часть поступающих в вычислительную систему данных вредоносны (представляют угрозу для системы) и могут быть обнаружены применяемыми в ней элементами ЗИ $(W=0.1)$, а также пусть $I_{S}=1$ (все применяемые средства в составе системы ЗИ покрывают все имеющиеся угрозы ИБ, направленные против рассматриваемой системы). Среднее время обслуживания каждым элементом системы ЗИ: $V_{1}=0.0025 \mathrm{c}$, $V_{2}=0.004 \mathrm{c}, V_{3}=0.0075$ с. Вероятность устранения угрозы ИБ каждым элементом системы ЗИ: $p_{1}=0.9$, $p_{2}=0.95, p_{3}=0.925$. Доли угроз ИБ от общего множества угроз ИБ, определяемых и устраняемых элементами системы ЗИ для рассматриваемых вариантов системы: $L_{1}=35 \%, L_{2}=50 \%, L_{3}=70 \%$, $l_{12.0}=15 \%, l_{13.0}=20 \%, l_{23.0}=30 \%, l_{123.0}=10 \%$. Допустимое время пребывания запроса в системе $T_{0}=0.2$ с. Стоимость каждого элемента защиты и ограничение на общую стоимость системы ЗИ $C_{0}=100$ y.e., $c_{1}=10$ y.e., $c_{2}=15$ y.e., $c_{3}=25$ y.e.

Эффективность системы ЗИ определим по комплексному критерию, учитывающему задержку и вероятность обнаружения и устранения угроз при последовательном применении средств ЗИ, вычисляемого по (1). Подставив в (1) формулы (5) и (7), получим показатель эффективности системы ЗИ, предполагая абсолютную надежность системы.

На рисунке 4 представлена зависимость эффективности системы ЗИ от интенсивности входного потока с различной комплектацией элементов ЗИ на вычислительном узле (4a) и систем, использующих разное количество элементов ЗИ при фиксированном количестве имеющихся в узле элементов защиты (4б).

Анализ зависимостей, представленных на графиках (рис. 4), показывает влияние состава и порядка последовательного применения средств ЗИ на эффективность системы ЗИ, а также существование границ эффективности применения вариантов комплектации и последовательности применения средств ЗИ.

При программной реализации средств ЗИ и их последовательной инициализации для обнаружения и устранения угроз возможна адаптивная стратегия предотвращения угроз ИБ в зависимости от измеряемой интенсивности поступающих запросов. При размещении в узлах защиты полного комплекта аппаратно-программных средств ЗИ становится потенциально реализуемой адаптация системы ЗИ к интенсивности поступающих запросов на основе оптимальной последовательности использования средств ЗИ. 


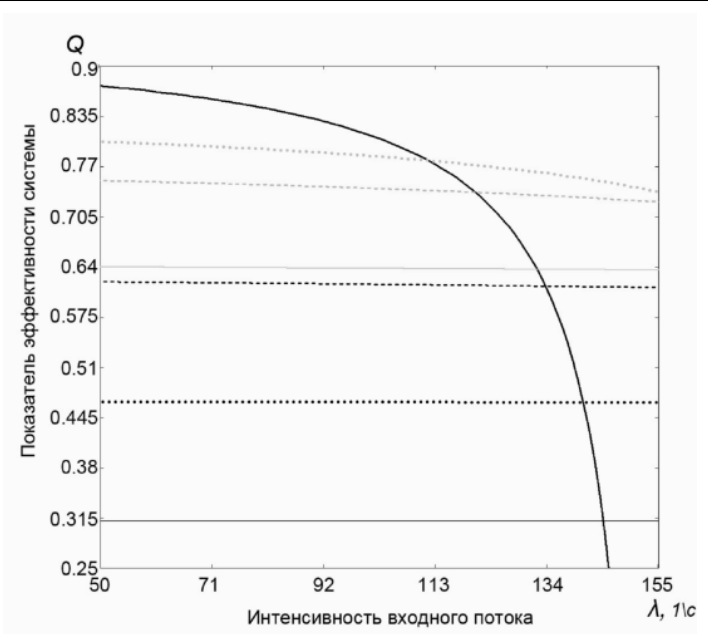

a)

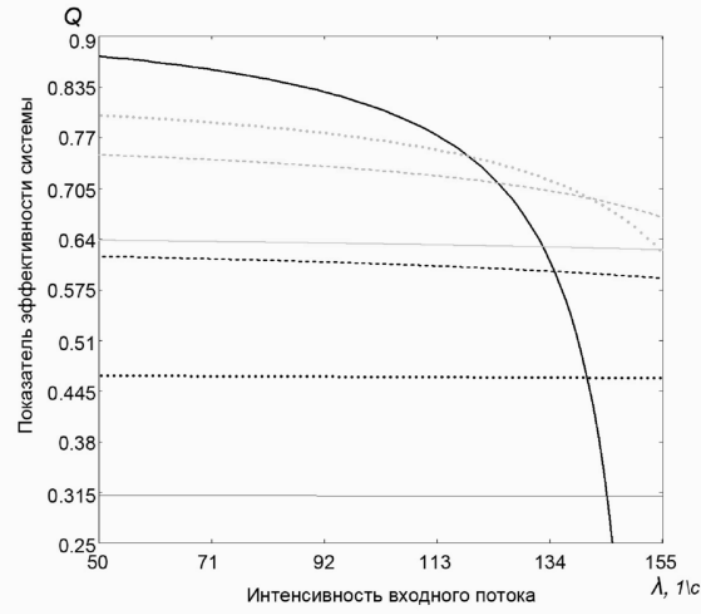

б)

Рис. 4. Зависимость эффективности системы ЗИ от интенсивности входного потока для систем с различным последовательным применением средств ЗИ. Вариант 1 - (1,2,3) (черная толстая линия); варианты 2-4-(1,2), (2,3), (1,3) (серая тонкая, штрихпунктирная, пунктирная линии, соответственно); варианты 5-7-(1), (2), (3) (серая тонкая, итрихпунктирная, пунктирная линии, соответственно)

Fig. 4. Dependence of information security system effectiveness on the intensity of the input flow for systems with different sequential use of means of information protection

При комплектации узлов защиты неполным набором реализуемых в виде аппаратно-программных средств ЗИ адаптивная настройка на оптимальную последовательность используемых средств ЗИ становится проблематичной. Целесообразность перекомпоновки (перекомплектации) узлов в зависимости от изменяющегося потока запросов требует дополнительных исследований, связанных с остановкой обслуживания при реконфигурации системы [14-17]. В случае неполной комплектации узлов аппаратно-программными средствами ЗИ при проектировании может решаться задача выбора оптимальной комплектации узлов средствами ЗИ и последовательности их применения, ориентируясь на ожидаемую (среднюю) интенсивность входного потока запросов, проверяемого на наличие угроз.

Повышение интенсивности входного потока нередко является следствием организации атаки на систему. Поэтому требуется установление минимальной конфигурации элементов ЗИ системы, которая будет способна обеспечить необходимый для системы уровень информационной защищенности при максимально возможном потоке запросов, формируемых при атаках [7, 18-23].

\section{Заключение}

Исследованы возможности повышения эффективности инфокоммуникационной системы, использующей СБД «Прямое соединение» при различных вариантах комплектации узлов средствами ЗИ и последовательности их применения при предотвращении угроз ИБ в зависимости от интенсивности входного потока запросов.

Предложены критерии эффективности систем ЗИ, ориентированные на повышение вероятности обнаружения угроз за минимальное время с учетом возможной ограниченности допустимых задержек запросов.

Предложены модели оценки вероятности обнаружения угроз и задержек их поиска в зависимости от последовательности применения средств ЗИ в узлах с учетом пересекаемости множеств угроз, обнаруживаемых различными средствами защиты.

Проанализированы возможность и целесообразность реализации адаптивной стратегии последовательного применения средств ЗИ для предотвращения угроз ИБ в зависимости от измеряемой интенсивности поступающих запросов при комплектации узлов полным набором программных или аппаратно-программных средств ЗИ.

\section{Литература}

1. Ellison R.J., Fisher D.A., Linger R.C., Lipson H.F., Longstaff T., Mead N.R. Survivable network systems: an emerging discipline. 1997. URL: http://www.cert.org $\mid$ research $\backslash 97$ tr013.pdf (дата обращения: 10.02.2018).

2. Korobeynikov A.G., Fedosovsky M.E., Maltseva N.K., Baranova O.V., Zharinov I.O., Gurjanov A.V., Zharinov O.O. Use of information technologies in design and production activities of instrument-making plants. Indian J. of Sc. and Tech., IET-2016, vol. 9 , no. 44 , pp. $1-8$.

3. Kopetz H. Real-time systems: design principles for distributed embedded applications. Springer, 2011, $396 \mathrm{p}$.

4. Советов Б.Я., Колбанев М.О., Татарникова Т.М. Технологии инфокоммуникации и их роль в обеспечении информационной безопасности // Геополитика и безопасность. 2014. № 1. C. $69-77$.

5. Коломойцев В.С. Выбор варианта построения многоуровневого защищенного доступа к внешней сети // Науч.-технич. вестн. информ. технологий, механики и оптики. 2016. T. 16. № 1. C. 115-121. 
6. Kleinrock L. Queueing system. Jogn Wiley \& Sons, Inc., 1975 , vol. $1,417 \mathrm{p}$

7. Коломойцев В.С., Богатырев В.А. Вероятностно-временные показатели при поэтапном применении средств защиты информации // Вестн. компьютер. и информ. технологий. 2017. № 11. C. 37-43. DOI: 10.14489/vkit.2017.11.pp. 037-043.

8. Sorin D. Fault tolerant computer architecture. Morgan \& Claypool, 2009, 103 p.

9. Кутузов О.И., Татарникова Т.М. Инфокоммуникационные сети. Моделирование и оценка вероятностно-временных характеристик. СПб: Изд-во ГУАП, 2015. 381 с.

10. Верзун Н.А., Колбанев М.О., Омельян А.В. Введение в инфокоммуникационные технологии и сети Future Networks. СПб: Изд-во СПбГЭУ, 2016. 51 с.

11. Верзун Н.А., Колбанев М.О., Татарникова Т.М. Технологическая платформа четвертой промышленной революции // Геополитика и безопасность. 2016. № 2. С. 73-78.

12. Богатырев В.А., Богатырев С.В. Резервированное обслуживание в кластерах с уничтожением неактуальных запросов // Вестн. компьютер. и информ. технологий. 2017. № 1 . C. $21-28$.

13. Богатырев В.А., Богатырев С.В. Критерии оптимальности многоустойчивых отказоустойчивых компьютерных систем // Науч.-технич. вестн. СПбГУ ИТМО. 2009. № 5. С. 92-98.

14. Колбанев М.О., Татарникова Т.М., Воробьев А.И. Оценка вероятностно-временных характеристик процесса предоставления информационно-справочных услуг // Изв. вузов: Приборостроение. 2014. Т. 57. № 9. С. 15-18.

15. Алиев Т.И., Муравьева-Витковская Л.А. Приоритетные стратегии управления трафиком в мультисервисных компьютерных сетях // Изв. вузов: Приборостроение. 2011. Т. 54. № 6. C. $44-48$.
16. Gatchin Y.A., Zharinov I.O., Korobeynikov A.G., Zharinov O.O. Theoretical estimation of Grassmann's transformation resolution in avionics color coding systems. Modern Applied Science, 2015, vol. 9, no. 5, pp. 197-210.

17. Korobeynikov A.G., Fedosovsky M.E., Gurjanov A.V., Zharinov I.O., Shukalov A.V. Development of conceptual modeling method to solve the tasks of computer-aided design of difficult technical complexes on the basis of category theory. Intern. J. of Applied Engineering Research, 2017, vol. 12, no. 6, pp. 1114-1122.

18. Гатчин Ю.А., Жаринов И.О., Коробейников А.Г. Математические модели оценки инфраструктуры системы защиты информации на предприятии // Науч.-технич. вестн. информ. технологий, механики и оптики. 2012. № 2. С. 92-95.

19. Щеглов К.А., Щеглов А.Ю. Метод реализации песочницы для потенциально опасных программ // Изв. вузов: Приборостроение. 2017. Т. 60. № 10. С. 940-944.

20. Kniga E., Zharinov I., Shukalov A., Nechaev V. Reliability evaluation of integrated modular avionics computational structures for different hardware configurations. Key Engineering Materials, 2016, vol. 685 , pp. 350-354. DOI: 10.4028/www.scientific.net/ KEM.685.350.

21. Колбанев М.О., Татарникова Т.М. Физические ресурсы информационных процессов и технологий // Науч.-технич. вестн. ИТМО. 2014. Т. 14. № 6. С. 113-123.

22. Nyrkov A.P., Goloskokov K.P., Koroleva E.A., Sokolov S.S., Zhilenkov A.A., Chernyi S.G. Mathematical models for solving problems of reliability maritime system. Lecture Notes in Electrical Engineering, 2018, vol. 442, pp. 387-394.

23. Nyrkov A.P., Sokolov S.S., Karpina A.S., Chernyakov A.V., Gaskarov V.D. Cluster-type models in the logistics industry. Intern. J. of Engineering and Technology (UAE), 2018, vol. 7 , no. 1 , pp. $39-43$.

\title{
The effectiveness of stage-by-stage use of securirty means crossing threat detection areas
}

\author{
V.S. Kolomoitsev ${ }^{1}$, Postgraduate Student, dek-s-kornis@yandex.ru \\ V.A. Bogatyrev ${ }^{1}$, D.Sc., Professor \\ ${ }^{1}$ The National Research University of Information Technologies, Mechanics and Optics, \\ St. Petersburg, 197101, Russian Federation
}

Abstract. The paper investigates the ways of improving the efficiency of a secure access scheme "direct connection". The scheme is based on choosing options for its construction, taking into account the phased use of various information security means and their integration into a cluster architecture system aimed at consolidating limited protection resources. Consolidation of protection resources is carried out in order to achieve maximum security with minimum delays and system costs.

The authors propose criteria for the effectiveness of information security systems aimed at increasing the probability of threat detection in the shortest time, taking into account its limitations. The proposed criteria take into account the average time of request stay in the system and the probability of threat detection and removal by an information security system. There is an assumption that the service time of each stage is a random value having exponential distribution.

The paper proposes models of estimating the probability of detection and elimination of threats and delays based on the sequence of application of information security means in the nodes. The models take into account intersections of sets of threats detected by various means of information security. The authors show the possibility of implementing an adaptive strategy of 
consistent application of information security means to prevent threats to information security depending on changing intensity of incoming requests. There are some options of bundling nodes by a complete or partial set of available software or hardwaresoftware means of information security.

Keywords: information protection, information security, access scheme, unauthorized access, system quality assessment, queueing system, probability of detecting threats, information threat.

\section{References}

1. Ellison R.J., Fisher D.A., Linger R.C., Lipson H.F., Longstaff T., Mead N.R. Survivable Network Systems: An Emerging Discipline. Software Engineering Institute. 1997. Available at: https://resources.sei.cmu.edu/asset_files/ technicalreport/1998_005_001_16598.pdf (accessed February 10,2018).

2. Korobeynikov A.G.G., Fedosovsky M.E., Maltseva N.K., Baranova O.V., Zharinov I.O., Gurjanov A.V., Zharinov O.O. Use of information technologies in design and production activities of instrument-making plants. Indian J. of Science and Technology. IET-2016, vol. 9, no. 44, pp. 1-8.

3. Kopetz H. Real-Time Systems: Design Principles for Distributed Embedded Applications. Springer Publ., 2011,396 p.

4. Sovetov B.Ya., Kolbanev M.O., Tatarnikova T.M. Technologies of infocommunication and their role in ensuring information security. Geopolitics and Security. 2014, no. 1, pp. $69-77$ (in Russ.).

5. Kolomoitsev V.S. Choice of option for implementation of the multilevel secure access to the external network. Sci. and Tech. J. of Information Technologies, Mechanics and Optics. 2016, vol. 16, no. 1, pp. 115-121 (in Russ.).

6. Kleinrock L. Queueing System. Vol. 1: Theory. J. Wiley \& Sons Publ., 1975, 417 p.

7. Kolomoitsev V.S., Bogatyrev V.A. Probabilistic and temporal indicators in the stage-by-stage use of information protection means. Herald of Computer and Information Technologies. 2017, no. 11, pp. 37-43 (in Russ.).

8. Sorin D. Fault Tolerant Computer Architecture. Morgan \& Claypool Publ., 2009, 103 p.

9. Kutuzov O.I., Tatarnikova T.M. Infocommunication Networks. Modeling and Estimation of Probability-Time Characteristics. St. Petersburg, SUAI Publ., 2015, 381 p.

10. Verzun N.A., Kolbanev M.O., Omelyan A.V. Introduction to Infocommunication Technologies and Future Networks. St. Petersburg, 2016, 51 p.

11. Verzun N.A., Kolbanev M.O., Tatarnikova T.M. A technological platform of the fourth industrial revolution. Geopolitics and Security. 2016, no. 2, pp. 73-78 (in Russ.).

12. Bogatyrev V.A., Bogatyrev S.V. Redundant maintenance in clusters with destruction of irrelevant requests. Herald of Computer and Information Technologies. 2017, no. 1, pp. 21-28 (in Russ.).

13. Bogatyrev V.A., Bogatyrev S.V. Optimality criteria of multistable fault-tolerant computer systems. Sci. and Tech. J. of Information Technologies, Mechanics and Optics. 2009, no. 5, pp. $92-98$ (in Russ.).

14. Kolbanev M.O., Tatarnikova T.M., Vorobev A.I. Assessment of probabilistic-temporal characteristics of the process of I\&R service rendering. J. of Instrument Engineering. 2014, vol. 57, no. 9, pp. 15-18 (in Russ.).

15. Aliev T.I., Muraveva-Vitkovskaya L.A. Priority-based strategies of traffic management in multiservice computer networks. J. of Instrument Engineering. 2011, vol. 54, no. 6, pp. 44-48 (in Russ.).

16. Gatchin Yu.A., Zharinov I.O., Korobeynikov A.G., Zharinov O.O. Theoretical estimation of Grassmann's transformation resolution in avionics color coding systems. Modern Applied Science. 2015, vol. 9, no. 5, pp. 197-210.

17. Korobeynikov A.G., Fedosovsky M.E., Gurjanov A.V., Zharinov I.O., Shukalov A.V. Development of conceptual modeling method to solve the tasks of computer-aided design of difficult technical complexes on the basis of category theory. Intern. J. of Applied Engineering Research. 2017, vol. 12, no. 6, pp. 1114-1122.

18. Gatchin Yu.A., Zharinov I.O., Korobeynikov A.G. Mathematical models of an estimation of an infrastructure of system of protection of the information at the enterprise. Sci. and Tech. J. of Information Technologies, Mechanics and Optics. 2012, no. 2, pp. 92-95 (in Russ.).

19. Shcheglov K.A., Shcheglov A.Yu. Implementation of sandbox method for potentially malicious applications. J. of Instrument Engineering. 2017, vol. 60, no. 10, pp. 940-944 (in Russ.).

20. Kniga E., Zharinov I., Shukalov A., Nechaev V. Reliability evaluation of integrated modular avionics computational structures for different hardware configurations. Key Engineering Materials. 2016, vol. 685, pp. 350-354.

21. Kolbanev M.O., Tatarnikova T.M. Physical resources of information processes and technologies. Sci. and Tech. J. of Information Technologies, Mechanics and Optics. 2014, vol. 14, no. 6, pp. 113-123 (in Russ.).

22. Nyrkov A.P., Goloskokov K.P., Koroleva E.A., Sokolov S.S., Zhilenkov A.A., Chernyi S.G. Mathematical models for solving problems of reliability maritime system. Lecture Notes in Electrical Engineering. 2018, vol. 442, pp. 387-394.

23. Nyrkov A.P., Sokolov S.S., Karpina A.S., Chernyakov A.V., Gaskarov V.D. Cluster-type models in the logistics industry. Intern. J. of Engineering and Technology (UAE). 2018, vol. 7, no. 1, pp. 39-43. 